\title{
BMJ Open Evaluating outpatient transition clinics: a mixed-methods study protocol
}

\author{
Jane N T Sattoe, ${ }^{1,2}$ Mariëlle A C Peeters, ${ }^{1,3}$ Sander R Hilberink, ${ }^{1}$ Erwin Ista, ${ }^{2}$ \\ AnneLoes van Staa ${ }^{1,3}$
}

To cite: Sattoe JNT, Peeters MAC, Hilberink SR, et al. Evaluating outpatient transition clinics: a mixedmethods study protocol. BMJ Open 2016;6:e011926. doi:10.1136/bmjopen-2016011926

- Prepublication history for this paper is available online. To view these files please visit the journal online (http://dx.doi.org/10.1136/ bmjopen-2016-011926).

Received 16 March 2016 Revised 13 July 2016 Accepted 26 July 2016

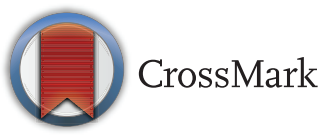

${ }^{1}$ Research Centre Innovations in Care, Rotterdam University of Applied Sciences, Rotterdam, The Netherlands ${ }^{2}$ Erasmus University Medical Center, Sophia Children's Hospital, Rotterdam, The Netherlands

${ }^{3}$ Institute of Health Policy \& Management, Erasmus University Rotterdam, Rotterdam, The Netherlands

Correspondence to Dr Jane N T Sattoe; j.n.t.sattoe@hr.nl

\section{ABSTRACT}

Introduction: To support young people in their transition to adulthood and transfer to adult care, a number of interventions have been developed. One particularly important intervention is the transition clinic (TC), where paediatric and adult providers collaborate. TCs are often advocated as best practices in transition care for young people with chronic conditions, but little is known about TC models and effects. The proposed study aims to gain insight into the added value of a TC compared with usual care (without a TC).

Methods and analysis: We propose a mixedmethods study with a retrospective controlled design consisting of semistructured interviews among healthcare professionals, observations of consultations with young people, chart reviews of young people transferred 2-4 years prior to data collection and questionnaires among the young people included in the chart reviews. Qualitative data will be analysed through thematic analysis and results will provide insights into structures and daily routines of TCs, and experienced barriers and facilitators in transitional care. Quantitatively, within-group differences on clinical outcomes and healthcare use will be studied over the four measurement moments. Subsequently, comparisons will be made between intervention and control groups on all outcomes at all measurement moments. Primary outcomes are "no-show after transfer' (process outcome) and 'experiences and satisfaction with the transfer' (patient-reported outcome). Secondary outcomes consider clinical outcomes, healthcare usage, self-management outcomes and perceived quality of care.

Ethics: The Medical Ethical Committee of the Erasmus Medical Centre approved the study protocol (MEC-2014-246).

Dissemination: Study results will be disseminated through peer-reviewed journals and conferences. The study started in September 2014 and will continue until December 2016. The same study design will be used in a national study in 20 diabetes settings (2016-2018).

\section{BACKGROUND}

Successful transfer from paediatric to adult healthcare services is a crucial aspect of high-quality care, but also forms a major

\section{Strengths and limitations of this study}

- The proposed study includes a controlled mixedmethods evaluation of process indicators, clinical and patient-reported outcomes. As such, it provides insights into the application and effects of a complex intervention.

- Based on the criteria of successful transition and an extensive literature research, we link possible outcomes to existing theory about the transition of young people with chronic conditions. This contributes to the development of a relevant evaluation framework for transitional care. In addition, we provide an approach that allows for comparisons between studies and even between disease groups.

- Some challenges associated with the design include the selection of control departments, response rates to the questionnaire and organisational issues around the chart reviews, particularly in case of transfer to other hospitals.

challenge for young people with chronic conditions. ${ }^{1}$ The gap between paediatric and adult healthcare services appears to be large. ${ }^{2}$ Studies showed that up to $25 \%$ of the young adults that have been transferred to adult healthcare services do not regularly attend follow-up visits in the hospital. ${ }^{34}$ Loss to follow-up may be a result of differences between paediatric and adult healthcare settings and a poor preparation for the transfer, but may also be affected by changes in social relationships and shifting roles. ${ }^{5}$ While adolescents become increasingly responsible for their own health, they generally show poor treatment adherence, which puts them at risk for poor health outcomes ${ }^{6}$ (eg, deterioration of lung function in cystic fibrosis $(\mathrm{CF})$ ). They value being seen as a competent partner in care, but too often feel that they are not included in important decisions about their own lives. ${ }^{25} 78$

To support young people in their transition to adulthood and transfer to adult care, a number of interventions have been developed and implemented..$^{9-11}$ One particularly 
relevant intervention is the transition clinic (TC). TCs are often advocated as best practice in transition care for young people with chronic conditions. ${ }^{9}{ }^{12-14}$ Although there is no common definition of a TC, the core principle is that professionals from paediatric and adult care are involved in the delivery of outpatient care in preparation for the upcoming transfer. ${ }^{915}$ While some TCs are focused on organising a smooth transfer to adult care and on good clinical outcomes, ${ }^{16}$ others have a broader focus including the transition to adulthood and associated psychosocial outcomes. ${ }^{17}$ Daily routines and used protocols differ considerably between TCs, ${ }^{18}$ and current literature lacks systematic descriptions of TCs' structures and working mechanisms. ${ }^{9}$ In addition, little is known about the effects of this intervention. A review in 2011 identified four evaluation studies of TCs, but these were all diabetes oriented. ${ }^{9}$ Although these studies found positive short-term health outcomes (better glycosylated haemoglobin (Hb1Ac) levels and less short-term complications) and follow-up rates, there is currently no evidence for (long-term) outcomes of TCs with regard to health outcomes, healthcare use, self-management and psychosocial functioning of young adults. Moreover, recent studies of TCs in other diagnostic groups did not include a controlled pre-post outcome evaluation, ${ }^{19-30}$ and as such could not provide sound evidence on outcomes of TCs.

In general, there is inconclusive evidence considering the effectiveness of transitional care. Also, although a large part of transition is generic and not disease specific, comparisons between disease groups and more general evaluations of interventions are rarely conducted. As paediatric or young adult diagnostic groups are often small, including more groups could be beneficial for effectiveness research. Moreover, a noncategorical approach would allow professionals of different disciplines to learn from each other. ${ }^{11}$ The recently formulated consensus indicators for successful transition $^{31}$ could be helpful for such comparisons and more generic evaluations. Furthermore, little information is provided about important elements of interventions used in transitional care, ${ }^{10}{ }^{32}$ and there is still little research into young people's and healthcare professionals' experiences with such interventions. ${ }^{433} 34$

\section{METHODS AND ANALYSIS}

\section{Study aim}

The proposed study evaluates the experiences with and outcomes of TCs. It aims to gain insight into the added value of a TC compared with usual care (without a TC) along the following research questions:

1. How do structures and daily routines differ between TCs and usual care?

2. What are healthcare professionals' experienced barriers and facilitators for the organisation of a TC?

3. What are the differences in clinical outcomes and healthcare use of young people who were seen at a $\mathrm{TC}$ and those that received usual care?
4. What are the differences in self-management outcomes, experiences and satisfaction with the transfer to adult care and perceived quality of care of young people who were seen at a TC and those that received usual care?

5. What are the differences between TCs and usual care with respect to the criteria for successful transition? ${ }^{31}$

\section{Study design}

A TC is a complex intervention that cannot easily be evaluated through a randomised controlled trial. It is important to explore how complex interventions form a part of and work within their contexts, so that possible working mechanisms could be revealed and the eventual outcomes could be attributed to the intervention. ${ }^{35}$ Therefore, the use of a mixed-methods design is advocated in the evaluation of complex interventions. ${ }^{36}{ }^{37} \mathrm{To}$ answer the research questions, a mixed-methods study with a retrospective controlled design will be used. The TC will be compared with usual care, that is, transfer to adult care without the use of a TC. Elements studied considering the formats of transitional care will be as follows: TC setting, availability of a written transfer, involved professionals, age group seen at TC, number of TC visits young people have before transfer, presence of dedicated professionals in adult care, structure and content of the TC consultations (including subjects discussed), and use of other interventions to support transition such as individual transition plans.

The qualitative part of this study will be conducted first and is expected to provide insight into the development, underlying thoughts, organisation, structure, team, facilitators and barriers of transitional care, both in the form of a TC and as standard care (without TC). Two data collection methods will be employed: observations during consultations with young people and semistructured interviews with healthcare professionals. The quantitative part explores young people's experiences with transitional care and clinical, healthcare and selfmanagement outcomes among those who received care at a TC and those who did not. Data collection methods will include retrospective chart review and survey among young adults transferred to adult care. An overview of the data collection methods per research question is presented in table 1.

\section{Study setting}

Purposive sampling will be used to select TCs in the Erasmus University Medical Center-Sophia Children's Hospital in Rotterdam. Previous research provided an overview of departments that have a TC in Erasmus University Medical Center. ${ }^{38}$ TCs operating for 4 years or longer will be selected and invited to participate, because these are expected to have more or less embedded and standardised TC structures and routines. Also, selecting longer existing TCs would allow for larger study samples, because a larger number of young people would be treated at the TCs over time. For each 
Table 1 Data collection methods per research question

\begin{tabular}{|c|c|c|}
\hline \multirow[b]{2}{*}{ Research questions } & \multicolumn{2}{|l|}{ Data collection method } \\
\hline & Quantitative & Qualitative \\
\hline $\begin{array}{l}\text { 1. How do structures and daily routines differ } \\
\text { between TCs and usual care? }\end{array}$ & Not applicable & $\begin{array}{l}\text { Observations of healthcare practice } \\
\text { and semistructured interviews with } \\
\text { healthcare professionals }\end{array}$ \\
\hline $\begin{array}{l}\text { 2. What are healthcare professionals' } \\
\text { experienced barriers and facilitators for the } \\
\text { organisation of a TC? }\end{array}$ & Not applicable & $\begin{array}{l}\text { Observations of healthcare practice } \\
\text { and semistructured interviews with } \\
\text { healthcare professionals }\end{array}$ \\
\hline $\begin{array}{l}\text { 3. What are the differences in clinical } \\
\text { outcomes and healthcare use of young } \\
\text { people who were seen at a TC and those } \\
\text { that received usual care? }\end{array}$ & $\begin{array}{l}\text { Retrospective chart reviews of } \\
\text { young people's medical records }\end{array}$ & Not applicable \\
\hline $\begin{array}{l}\text { 4. What are the differences in } \\
\text { self-management outcomes, experiences } \\
\text { and satisfaction with the transfer to adult } \\
\text { care, and perceived quality of care of young } \\
\text { people who were seen at a TC and those } \\
\text { that received usual care? }\end{array}$ & Survey among young people & Not applicable \\
\hline $\begin{array}{l}\text { 5. What are the differences between TCs and } \\
\text { regular transition care on the criteria for } \\
\text { successful transition? }\end{array}$ & $\begin{array}{l}\text { Retrospective chart review of young } \\
\text { people's medical records and } \\
\text { survey among young people }\end{array}$ & Not applicable \\
\hline
\end{tabular}

participating TC, a control setting that provides usual care to the same diagnostic groups in one of the other University Medical Centres in the Netherlands will be selected. All departments will be contacted by email to inform them about the study and to ask for their cooperation, followed by a phone call (in case of no reply). After consent, two researchers will visit the centres to explain the study in more detail. The teams will then be asked for suggestions for control settings that can be contacted to ask for participation.

Based on the selection criteria and the previous overview of TCs in the Erasmus $\mathrm{MC}^{38}$ two pulmonology departments treating CF, two gastroenterology departments treating inflammatory bowel disease (IBD) (Crohn's disease and ulcerative colitis) and two endocrinology departments treating Turner syndrome (TS), congenital adrenal hyperplasia (CAH) and hypopituitarism will be invited to participate in the study.

\section{Study procedures}

Observations

Participant observations will be conducted at the TCs and at the outpatient control clinics. The aim is to observe about 8 hours in total at each setting. Young people and their parents will receive information about this study from their healthcare professional and all parties involved will be asked to provide written consent to the presence of the observer during their consultations. At the TCs, the consultations between young people, their parents and healthcare professionals will be observed, as well as the preparation of the professionals. In the control settings, regular consultations with young people and their parents will be observed. Attention will be paid to different themes including coordination of the transition process, structure, content of consultations and use of interventions. The researchers will take field notes and write down their findings in narratives.

\section{Semistructured interviews}

Healthcare professionals from paediatric and adult care, working at all participating departments will be invited for semistructured interviews by obtaining their email addresses through the departments' heads, who are asked to inform their teams about the study on beforehand. Non-responders will be reminded by email or a telephone call. Professionals from all relevant disciplines that participate in transitional care (eg, doctors, nurses, psychologists, dieticians, social workers, physiotherapists, etc) will be included.

Themes that will be addressed during the interviews are based on the literature, such as the validated 'You're welcome' quality criteria that determine whether a clinic can be typified young people-friendly, ${ }^{39} 40$ the "Mind the Gap' tool that is used to assess transfer readiness, ${ }^{41}$ and experiences of young adults, parents and profes$\begin{array}{lllllll}\text { sionals. }^{2} & 5 & 7 & 8 & 42 & 43 & \text { Examples of topics are as follows: }\end{array}$ reasons for (not) setting up a $\mathrm{TC}$, usefulness of the $\mathrm{TC}$, barriers and facilitators, coordination of the $\mathrm{TC}$, structure and content of transitional care and changes over time, involved healthcare professionals, use of interventions and added value of the TC for young people, their parents and healthcare professionals. Interviews will last for $\sim 1$ hour.

\section{Retrospective chart review and survey}

Of each outpatient department, all patients who have transferred to adult care 2-4 years prior to data 
collection will be selected for retrospective chart review and the survey. In case a patient has been transferred to adult care in another hospital, the clinician of the children's hospital will obtain contact details. Patients with severe intellectual disabilities or known psychiatric problems will be excluded. Information about such problems will be derived from the patient charts. An information letter accompanied by an invitation for participation in the survey and a consent form will be sent to all selected patients. A reminder letter will be sent to non-responders after 2 weeks, followed by a telephone call after 4 weeks. The survey will be sent to all patients that provided consent after chart review. For the chart review, both data from paediatric and adult care will be collected at four measurement moments: T-2, the second year before transfer; T-1, the year before transfer; $\mathrm{T} 1$, the year after transfer; and T2, the second year after transfer.

\section{Process and outcome measures for chart review and survey}

Although there are no agreed outcome measures for 'successful transition', several studies and study protocols for the evaluation of transitional care provide insights in important areas of outcome and process measures. ${ }^{34}$ 44-46 These include clinical outcomes, healthcare-related measures (medical follow-up) and psychosocial outcomes. Also, a recent international Delphi study provided insight into key indicators for successful transition, ${ }^{31}$ while another one recently identified key transition outcomes. ${ }^{47}$ We differentiate between process and (patient-reported) outcome measures.

Since 'no-show after transfer' is seen as an important process measure for transitional care, ${ }^{73145}$ it is selected as a primary process outcome in this study. Information about no-show will be derived from the patient charts. In the chart review, the following secondary measures will be assessed: clinical outcomes, number of consultations and hospital admissions, therapeutic regimen, and if available quality of life. Specific measures are based on previous research (see references in the tables) and were discussed with professionals working in the specific fields of endocrinology, CF and IBD.

With respect to the outcome measures retrieved through the survey, young people's 'experiences and satisfaction with transfer' will be considered a primary patient-reported outcome in this study. In the survey, the following secondary outcomes will be assessed: healthcare-related and self-management outcomes, experiences with current care and quality of life. These outcome measures are based on literature around transfer of young people, both disease-specific and more generic (see references in the tables).

We categorised the variables into background, process and outcome variables. Table 2 presents an overview of relevant background variables to be collected; table 3 explains the operationalisation of included process measures; and table 4 elaborates on the operationalisation of the outcome measures.

\section{Table 2 Relevant background variables}

\begin{tabular}{|c|c|c|}
\hline Variables & Operationalisation & $\begin{array}{l}\text { Data collection } \\
\text { method }\end{array}$ \\
\hline Date of birth & dd/mm/yyyy & Chart review \\
\hline $\begin{array}{l}\text { Date of } \\
\text { diagnosis }\end{array}$ & $\mathrm{dd} / \mathrm{mm} /$ yyyy & Chart review \\
\hline Gender & Male/female & Chart review \\
\hline Comorbidity & Yes/No & Chart review \\
\hline $\begin{array}{l}\text { Educational } \\
\text { level }\end{array}$ & High/medium/low* & Survey \\
\hline $\begin{array}{l}\text { Type of } \\
\text { education }\end{array}$ & $\begin{array}{l}\text { Special education or } \\
\text { not }\end{array}$ & Survey \\
\hline $\begin{array}{l}\text { Disability } \\
\text { benefits }\end{array}$ & Yes/No & Survey \\
\hline
\end{tabular}

\section{Data analyses}

\section{Qualitative analyses}

Interviews are audio taped, transcribed verbatim and imported into Atlas.ti 7.0. Interview transcripts and observation narratives will be coded thematically by two researchers independently. Themes will be derived from the topic lists used during the interviews, and if applicable, subthemes will be derived from the data. ${ }^{86}$ Details will be collected on the contents of the intervention, structure and working ways, and possible changes over time. Furthermore, healthcare professionals' experiences with transitional care, as well as experienced facilitators and barriers will be explored. As such, these analyses will provide answers on the first two research questions.

\section{Quantitative analyses}

First within-group differences on clinical outcomes and healthcare use will be studied over the four measurement moments with analysis of variance (ANOVA) tests (chart review data). Subsequently, comparisons will be made between intervention and control groups on clinical outcomes, healthcare use, self-management outcomes, experiences and satisfaction with the transfer to adult care and the perceived quality of current care. Independent samples t-tests and $\chi^{2}$ tests will be used to do so. These analyses will provide answers on the third and fourth research questions. The fifth research question is based on criteria for successful transition. To establish these, we used the recently established indicators of successful transition. ${ }^{31}$ The indicators or criteria that reached international consensus on being essential or very important for a successful transition and our operationalisation and data collection method per criteria are presented in table 5. Again, comparisons will be made between intervention and control groups with $\chi^{2}$ tests. Data per diagnostic group will be analysed. For the overall analysis of the generic outcomes (all but the clinical outcomes), all data will be compiled. Quantitative analyses will be performed with IBM SPSS 21.0. 
Table 3 Operationalisation of process measures

\begin{tabular}{|c|c|c|c|c|}
\hline Area* $^{*}$ & Variable & Operationalisation & $\begin{array}{l}\text { Measurement } \\
\text { moment } †\end{array}$ & $\begin{array}{l}\text { Data } \\
\text { collection } \\
\text { method }\end{array}$ \\
\hline \multirow[t]{4}{*}{ Transfer } & Date of transfer & dd/mm/yyyy & T0 & Chart review \\
\hline & Availability written transfer & Yes/No & T0 & Chart review \\
\hline & Written transfer recipient & Description & T0 & Chart review \\
\hline & $\begin{array}{l}\text { Current healthcare } \\
\text { provider }\end{array}$ & Centre & T0 & Chart review \\
\hline \multirow[t]{8}{*}{$\begin{array}{l}\text { Medical } \\
\text { follow-up } 37314548-61\end{array}$} & $\begin{array}{l}\text { No-show at first } \\
\text { appointment in adult care } \neq\end{array}$ & Yes/No & $\mathrm{T} 1$ & Chart review \\
\hline & $\begin{array}{l}\text { First appointment in adult } \\
\text { care cancelled }\end{array}$ & Yes/No & $\mathrm{T} 1$ & Chart review \\
\hline & Scheduled consultations & Number per year & $\mathrm{T}-2, \mathrm{~T}-1, \mathrm{~T} 1, \mathrm{~T} 2$ & Chart review \\
\hline & Missed consultations & Number per year & $\mathrm{T}-2, \mathrm{~T}-1, \mathrm{~T} 1, \mathrm{~T} 2$ & Chart review \\
\hline & $\begin{array}{l}\text { Time between last } \\
\text { appointment in paediatric } \\
\text { care and first appointment } \\
\text { in adult care }\end{array}$ & Months & $\mathrm{T}-1, \mathrm{~T} 1$ & Chart review \\
\hline & $\begin{array}{l}\text { Hospitalisations related to } \\
\text { chronic condition }\end{array}$ & Number per year & $\mathrm{T}-2, \mathrm{~T}-1, \mathrm{~T} 1, \mathrm{~T} 2$ & Chart review \\
\hline & Length of hospitalisations & Days & $\mathrm{T}-2, \mathrm{~T}-1, \mathrm{~T} 1, \mathrm{~T} 2$ & Chart review \\
\hline & $\begin{array}{l}\text { Emergency department } \\
\text { visits }\end{array}$ & Number per year & $\mathrm{T}-2, \mathrm{~T}-1, \mathrm{~T} 1, \mathrm{~T} 2$ & Chart review \\
\hline Healthcare & $\begin{array}{l}\text { Topics discussed during } \\
\text { consultations }\end{array}$ & $\begin{array}{l}\text { The need for more attention for } \\
\text { discussion of non-medical issues, } \\
\text { and the frequency of communication } \\
\text { about these topics during } \\
\text { consultations }\end{array}$ & Not applicable & Survey \\
\hline
\end{tabular}

Sample size: In an IBD sample of young people, it was found that $78 \%$ of the young people who were directly transferred versus $29 \%$ of those transferred through a TC had at least one recorded non-attendance at clinic after transfer. ${ }^{19}$ Since attending scheduled visits in adult care is an indicator for a successful transition, ${ }^{31}$ and no other studies provided relevant information on any of the indicators, we performed a sample size calculation based on this indicator and the results found by Cole and colleagues. Based on their numbers, an $\alpha$ of 0.05 and a power of 0.95 , we calculated that in the intervention and control group, the sample size should be 72 or more. Sample size calculation was performed with G*Power 3.2.1.

\section{ETHICS AND DISSEMINATION}

To ensure data confidentiality, the following procedures will be followed. Patients' personal identification information will only be available to the healthcare team and two researchers who are not part of the healthcare team. These researchers will sign a non-disclosure agreement (NDA). Other researchers will receive anonymised data. Patient numbers will be secured with passwords known by the researchers that signed the NDA and one member of the healthcare team. Young people and their parents (in case of minors who can be included in the observation part of the study) will receive an information letter from the doctor who is in charge of the treatment. They will be asked to provide written informed consent per study part. They are also asked to provide consent for matching the data from the surveys and chart reviews. Data will be processed anonymously and respondent numbers will be used to link data from the chart review to data from the survey. If applicable, pseudonyms will be used in the interview transcripts and observation narratives. The Medical Ethical Committee of the Erasmus Medical Centre approved the study protocol (MEC-2014-246). Study results will be published in international peer-reviewed journals, and will be presented at national and international conferences. The study started in September 2014 and is anticipated to continue until December 2016. The same study design will be used in a national study in 20 diabetes settings, starting in April 2016 until 2018.

\section{DISCUSSION}

Structuring the transition process by means of a TC is advocated to organise collaboration between paediatric 


\section{Area*}

Variable

Clinical outcomes in cystic

fibrosis $^{58-60} 62-65$

Clinical outcomes in

$\mathrm{IBD}^{51} 5666$

Clinical outcomes in endocrinology 5767

Healthcare-related

outcomes

Self-management-related

outcomes

\section{Pulmonary functioning}

Acute pulmonary exacerbations

Pseudomonas infection

Body mass index

Surgical treatments

Medications

Endoscopies

Body mass index

Blood pressure

For those with TS: thyroid function

For those with $\mathrm{CAH}$ : androgens

For those with hypopituitarism:

testosterone level

For those with hypopituitarism: insulin-like growth factor

For those with hypopituitarism: FT4 level

Medications

Experiences and satisfaction with transition to adult

care $^{732} 445253556066$ 68-72

Perceived quality of

care $^{732445253556066 \text { 68-72 }}$

Self-management ${ }^{11}$

Independence during consultations ${ }^{77}$

Self-efficacy $^{7465153616670717879}$

\section{Operationalisation}

FEV1 value

Use of antibiotics: yes/no, and frequency

Yes/No

Value

Number per year

Use and type

Number per year

Value

Systolic and diastolic

TSH/FT4 value

17-OHP/androstenedione value

Value

IGF-1 level

Value

Use and type

Experiences on two domains: (1) organisation of healthcare related to transition and (2) satisfaction with preparation for transfer

Patient centeredness on five domains: (1) empowerment, (2) design of practice, (3) goal-setting/alignment, (4) problem-solving and (5) coordination/ follow-up

Chronic condition self-management

Self-reported independent behaviours during consultations with healthcare providers

Disease-related self-efficacy on fou domains: (1) knowledge about the condition, (2) coping, (3) competencies
Measurement moment† (for chart review data) or measurement scale and psychometrics (for survey data)

$\mathrm{T}-2, \mathrm{~T}-1, \mathrm{~T} 1, \mathrm{~T} 2$

$\mathrm{T}-2, \mathrm{~T}-1, \mathrm{~T} 1, \mathrm{~T} 2$

$\mathrm{T}-2, \mathrm{~T}-1, \mathrm{~T} 1, \mathrm{~T} 2$

$\mathrm{T}-2, \mathrm{~T}-1, \mathrm{~T} 1, \mathrm{~T} 2$

$\mathrm{T}-2, \mathrm{~T}-1, \mathrm{~T} 1, \mathrm{~T} 2$

$\mathrm{T}-2, \mathrm{~T}-1, \mathrm{~T} 1, \mathrm{~T} 2$

$\mathrm{T}-2, \mathrm{~T}-1, \mathrm{~T} 1, \mathrm{~T} 2$

$\mathrm{T}-2, \mathrm{~T}-1, \mathrm{~T} 1, \mathrm{~T} 2$

$\mathrm{T}-2, \mathrm{~T}-1, \mathrm{~T} 1, \mathrm{~T} 2$

$\mathrm{T}-2, \mathrm{~T}-1, \mathrm{~T} 1, \mathrm{~T} 2$

$\mathrm{T}-2, \mathrm{~T}-1, \mathrm{~T} 1, \mathrm{~T} 2$

$\mathrm{T}-2, \mathrm{~T}-1, \mathrm{~T} 1, \mathrm{~T} 2$

$\mathrm{T}-2, \mathrm{~T}-1, \mathrm{~T} 1, \mathrm{~T} 2$

Data collection method

Chart review

Chart review

Chart review

Chart review

Chart review

Chart review

Chart review

Chart review

Chart review

Chart review

Chart review

Chart review

Chart review

$\mathrm{T}-2, \mathrm{~T}-1, \mathrm{~T} 1, \mathrm{~T} 2$

$\mathrm{T}-2, \mathrm{~T}-1, \mathrm{~T} 1, \mathrm{~T} 2$

On Your Own Feet Transition

Experience Scale (OYOF-TES) ${ }^{4}$

(validated 18-item scale with 5-point

Likert scales, $\alpha=0.92)+$ self-reported satisfaction on a $1-10$ scale

Patient Assessment of Chronic Illness

Care (PACIC) (validated 20-item scale with 5-point Likert scales) ${ }^{73} 74$

Partners in Health Scale (PIH)

Survey

(validated 12-item scale with 9-point

Likert scales) 7576

Independent Behaviours During

Consultations (IBDC) (validated 7-item scale with 5 -point Likert scales, $\alpha=0.79)^{77}+$ self-reported

independence on a 1-10 scale

On Your Own Feet Self-efficacy Scale (OYOF-SES) (validated 16-item scale with 4-point Likert scales) ${ }^{80}$ 


\section{Table 4 Continued}

Area*

Variable

Adherence $^{751-545658628182}$

Quality of life $44-4651-535862636768718184$
Operationalisation

during consultations and (4)

\section{medication}

Self-reported adherence to medical

treatment

Health-related quality of life on four domains: (1) physical, (2) emotional, (3) social and (4) school/work
Measurement moment† (for chart review data) or measurement scale and psychometrics (for survey data)

method

Medication Adherence Rating Scale

Survey

(MARS-5) (validated 5-item scale with 5-point Likert scales) (R Horne, M Hankins. The Medication Adherence

Report Scale (MARS): a new measurement tool for eliciting patients' reports of non-adherence.

Unpublished Working paper 2007) ${ }^{83}+$ self-reported adherence on a 1-10 scale

Paediatric Quality of Life Inventory

Young Adult (PedsQL_YA) (validated

3-item scale with 5-point Likert scales) $)^{85}$

${ }^{*}$ References include general and disease-specific studies that included and/or recommended similar outcome measures to study transition.

TT-2: the second year before transfer; T-1: the year before transfer; T0: transfer; T1: the year after transfer; and T2: the second year after transfer.

TT-2: the second yeal

17-OHP, 17-hydroxyprgesterone; CAH, congenital adrenal hyperplasia; FEV1, forced expiratory volume in 1 s; FT4, free thyroxine; IBD, inflammatory bowel disease; IGF-1, insulin-like growth factor-1; TS, Turner syndrome; TSH, thyroid-stimulating hormone. 
Table 5 Operationalisation of the criteria for successful transition ${ }^{31}$

Original criteria

1 Patient not lost to follow-up

2 Attending scheduled visits in adult care

3 Patient building a trusting relationship with adult provider

4 Continuing attention for self-management

5 Patient's first visit in adult care no later than 3-6 months after transfer

6 Number of emergency room visits for regular care in the past year

7 Patient and family satisfaction with transfer of care

8 Maintain/improvement of standard for disease control
Operationalisation: successful transfer if...

Young adult attended first planned consultation in adult care: yes/no

Young adult has no missed consultations in the 2 years after transfer: yes/no

Young people trust their current healthcare provider: a score higher than 6 points on a 10-point scale

There is sufficient attention for self-management (including non-medical) topics: average score of 3 or higher on 5-point scale

The first consultation in adult care takes place within

6 months after transfer: yes/no

There were no emergency room visits due to acute disruption of the disease in the 2 years after transfer: yes/no

Young people are satisfied with the transfer process: a score higher than 6 points on a 10-point scale

The standard for disease control evaluation is met in the year after transfer: yes/no and adult care and for better preparation of all parties involved..$^{9}$ 12-14 Apparently, this recommendation has been taken up by the field. While Crowley and colleagues found only four evaluation studies of TCs in 2011, ${ }^{9}$ there has been a marked increase in the publication of evaluation studies of TCs since 2015. We found new studies in the fields of epilepsy, urology, diabetes, CF, IBD, kidney disease, HIV and rheumatology. ${ }^{19-30} 8788$ Still, the proposed study is unique in its design. First, because only two published studies included a historical control group in the study design. ${ }^{19}{ }^{24}$ Our approach goes even further by including similar controls in the same time frame, adding the patient questionnaire and employing a pre-post design.

Second, our proposed design includes a significant qualitative study part that is expected to provide important insights into the daily routines, structures and working elements of TCs. Only one existing study employs qualitative data collection methods. Still, this study included a prospective evaluation among young adults $^{29}$ and therefore provides no insights into the TC model. Therefore, it is still unclear what TC models are implemented and what the best model might be. ${ }^{15} 89$ Our study could provide some answers to this question since we study components and outcomes of different TC models employing a standardised evaluation framework and the same data collection methods, which enables comparisons.

Finally, the evaluation studies we found vary in the outcome measures they use to evaluate the TC. While almost all evaluate whether or not young people attend adult care after transfer, some are interested in young people's satisfaction with the care delivered and programme components, ${ }^{26} 2888$ and others look at diseasespecific outcomes or adherence. ${ }^{19} 22242987$ Still, none of the studies clarify why certain outcome measures were chosen. We selected our outcome measures after extensive literature research, linking possible outcomes to current ideas about the transition of young people with chronic conditions. By doing so, we provide a relevant approach allowing for comparisons of transitional care between disease groups (eg, based on the criteria for successful transition ${ }^{31}$ ).

There are some challenges associated with our study design that need to be addressed. First, the current lack of insight into different TC models complicates the selection of outcome measures for the quantitative evaluation. We are not able to select these based on the content of the interventions. However, we view transition in the light of the definition proposed by Blum and colleagues in 1993 as 'a multifaceted, active process that attends to the medical, psychosocial, and educational/ vocational needs of adolescents as they move from the child-focused to the adult-focused health-care system', and as such transition 'implies an increase in independent behaviour and personal autonomy'. ${ }^{90}$ They further stated that 'the optimal goal of transition is to provide health care that is uninterrupted, coordinated, developmentally appropriate, psychosocially sound, and comprehensive'. ${ }^{90}$ Starting from this point of view, we conducted an extensive literature study and selected outcome measures that reflect these goals. Although not all TC models may include elements aimed at improving the whole transition process, we believe the selection of a wide variety of outcome measures may enable the comparisons of different TC models.

The second challenge, in connection with the lack of insight in TC models, considers the selection of control departments. The core principle of TCs is that healthcare professionals from paediatric and adult care are involved in the delivery of outpatient care in preparation for the upcoming transfer. However, the focus of this 
care (eg, medical and/or psychosocial needs) differs between TCs. This means that when defining usual care, that is, selecting control departments, we can only select on whether or not professionals from paediatric and adult care actively collaborate in the delivery of transitional care. It might be that even in the control setting some sort of collaboration is established, diluting the differences between usual care and care at a TC. To cope with this issue, we will also conduct qualitative research at these control sites. As such, we can specifically define what usual care in the control departments entails and how this differs from care in the intervention departments.

Furthermore, the retrospective character of the quantitative data might be a limitation. As participants will be asked to think about their transfer experiences from 2-4 years ago, this might lead to recall bias. Finally, the chart review and survey carry operational challenges with them. Most hospitals use different electronic patient record systems, and charts from 4 years ago may not even be digitalised yet. Also, there are usually very general formats within record systems, making it unclear whether or where information is available. To overcome these challenges, there will be close collaboration with at least one healthcare professional from each participating department. This professional will help us to draft a grid for chart review, and will explain their patient record system to us and show us where to find the required information. Since chart reviews include a very precise task, these will be conducted in teams of two researchers (who both will be instructed by the healthcare professional). Another challenge is the fact that it is not uncommon for young people to transfer to other hospitals, including some that may not be included in the study. This complicates the retrieval of post-transfer data for that group. Still, in the Netherlands, information from patient charts may be requested from the information department in the hospitals after written consent from the patient. Consent from young people who transferred to other hospitals will be sought. As for the survey, the response rate of young adults may be a problem. It is well known that response rates to questionnaires in adolescents and young adults are usually low. ${ }^{91} 92$ To anticipate this issue, all young people who fill out the questionnaire will be presented with a gift voucher of $€ 10$. Furthermore, parents are an important actor when it comes to transitional care, and it would be good to include them in the survey part. However, we anticipate difficulties in locating the parents of the young people, since we study a group of young adults transferred 2- 4 years ago. Therefore, we chose to focus on the young people alone.

Finally, the observations are current, while the quantitative evaluation is retrospective. This may complicate the use of the qualitative data from the observations to understand the outcomes of the evaluation. Still, we conduct qualitative interviews with professionals to gain insight into any changes that occurred in the working ways of the TC in the past 5 years. This will support the interpretation of the observational and quantitative data.

Contributors JNTS and AvS conceived of the study. All authors participated in its design. JNTS carried out the literature study and drafted the manuscript, while the others contributed to refinement. All authors have read and approved the final version.

Funding This study is part of the 'Self-Management and Participation Innovation Lab', supported by SIA-RAAK, the Foundation Innovation Alliance with funding from the Dutch Ministry of Education, Culture and Science (grant number PR0-02-025). The mentioned diabetes study is funded by 'Diabetes Fonds' (grant number 2015.30.1852) and 'Fonds NutsOhra' (grant number 101.325).

Disclaimer The funding sources had no role in the design and conduct of the study; collection, management, analysis and interpretation of the data; preparation, review and approval of the manuscript; and decision to submit the manuscript for publication.

Competing interests None declared.

Ethics approval The Medical Ethical Committee of the Erasmus Medical Centre.

Provenance and peer review Not commissioned; externally peer reviewed.

Data sharing statement Our manuscript describes a study protocol. As such, we cannot elaborate on additional unpublished data (yet).

Open Access This is an Open Access article distributed in accordance with the Creative Commons Attribution Non Commercial (CC BY-NC 4.0) license, which permits others to distribute, remix, adapt, build upon this work noncommercially, and license their derivative works on different terms, provided the original work is properly cited and the use is non-commercial. See: http:// creativecommons.org/licenses/by-nc/4.0/

\section{REFERENCES}

1. Viner RM. Transition of care from paediatric to adult services: one part of improved health services for adolescents. Arch Dis Child 2008;93:160-3.

2. van Staa AL, Jedeloo S, van Meeteren J, et al. Crossing the transition chasm: experiences and recommendations for improving transitional care of young adults, parents and providers. Child Care Health Dev 2011;37:821-32.

3. Downing J, Gleeson HK, Clayton PE, et al. Transition in endocrinology: the challenge of maintaining continuity. Clin Endocrinol (Oxf) 2013;78:29-35.

4. van Staa A, Sattoe JNT. Young adults' experiences and satisfaction with the transfer of care. J Adolesc Health 2014;55 796-803.

5. Fegran L, Hall EO, Uhrenfeldt L, et al. Adolescents' and young adults' transition experiences when transferring from paediatric to adult care: a qualitative metasynthesis. Int $J$ Nurs Stud 2014;51:123-35.

6. Sawyer SM, Drew S, Yeo MS, et al. Adolescents with a chronic condition: challenges living, challenges treating. Lancet 2007;369:1481-9.

7. Lugasi T, Achille M, Stevenson M. Patients' perspective on factors that facilitate transition from child-centered to adult-centered health care: a theory integrated metasummary of quantitative and qualitative studies. J Adolesc Health 2011;48:429-40.

8. Betz CL, Lobo ML, Nehring WM, et al. Voices not heard: a systematic review of adolescents' and emerging adults' perspectives of health care transition. Nurs Outlook 2013;61:311-36.

9. Crowley R, Wolfe I, Lock K, et al. Improving the transition between paediatric and adult healthcare: a systematic review. Arch Dis Child 2011;96:548-53.

10. Chu PY, Maslow GR, von Isenburg M, et al. Systematic review of the impact of transition interventions for adolescents with chronic illness on transfer from pediatric to adult healthcare. J Pediatr Nurs 2015;30:e19-27.

11. Sattoe JNT, Bal MI, Roelofs PD, et al. Self-management interventions for young people with chronic conditions: a systematic overview. Patient Educ Couns 2015;98:704-15.

12. Camfield P, Camfield C, Pohlmann-Eden B. Transition from pediatric to adult epilepsy care: a difficult process marked by medical and social crisis. Epilepsy Curr 2012;12:13-21. 
13. Davies IH, Jenkins HR. Transition clinics for adolescents with chronic gastrointestinal disease in the UK and Ireland. J Pediatr Gastroenterol Nutr 2003;36:505-6.

14. Viner R. Transition from paediatric to adult care. Bridging the gaps or passing the buck? Arch Dis Child 1999;81:271-5.

15. Carrizosa J, An I, Appleton R, et al. Models for transition clinics. Epilepsia 2014;55:46-51.

16. Betz CL, Redcay G. Lessons learned from providing transition services to adolescents with special health care needs. Issues Compr Pediatr Nurs 2002;25:129-49.

17. Falcini F, Nacci F. Systemic lupus erythematosus in the young: the importance of a transition clinic. Lupus 2007;16:613-17.

18. Iyer A, Appleton R. Transitional services for adolescents with epilepsy in the U.K.: a survey. Seizure 2013;22:433-7.

19. Cole R, Ashok D, Razack A, et al. Evaluation of outcomes in adolescent inflammatory bowel disease patients following transfer from pediatric to adult health care services: case for transition. $J$ Adolesc Health 2015;57:212-17.

20. Geerlings R, Aldenkamp A, Gottmer-Welschen L, et al. Evaluation of a multidisciplinary epilepsy transition clinic for adolescents. Eur J Paediatr Neurol 2016;20:385-92.

21. Mistry B, Van Blyderveen S, Punthakee Z, et al. Condition-related predictors of successful transition from paediatric to adult care among adolescents with Type 1 diabetes. Diabet Med 2015;32:881-5

22. Uday S, Campbell FM, Yong J, et al. Transition and beyond in adolescents and young adults with type 1 diabetes mellitus. Int $J$ Endocr Metab Disord 2015;1(2); doi:10.16966/2380-548X.106

23. Gravelle AM, Paone M, Davidson AGF, et al. Evaluation of a multidimensional cystic fibrosis transition program: a quality improvement initiative. J Pediatr Nurs 2015;30:236-43.

24. McQuillan RF, Toulany A, Kaufman M, et al. Benefits of a transfer clinic in adolescent and young adult kidney transplant patients. Can J Kidney Health Dis 2015;2:1-8.

25. Timberlake MD, Corbett ST, Costabile RA, et al. Identification of adolescent and adult patients receiving pediatric urologic care and establishment of a dedicated transition clinic. J Pediatr Urol 2015;11: e61-62.

26. Shalaby MS, Gibson A, Granitsiotis P, et al. Assessment of the introduction of an adolescent transition urology clinic using a validated questionnaire. J Pediatr Urol 2015;11:e81-89.

27. Maturo $\mathrm{D}$, Powell $\mathrm{A}$, Major-Wilson $\mathrm{H}$, et al. Transitioning adolescents and young adults with HIV infection to adult care: pilot testing the "Movin'Out" transitioning protocol. J Pediatr Nurs 2015;30:e29-35.

28. Stringer E, Scott R, Mosher D, et al. Evaluation of a rheumatology transition clinic. Pediatr Rheumatol 2015;13:22.

29. Tong A, Gow K, Wong G, et al. Patient perspectives of a young adult renal clinic: a mixed-methods evaluation. Nephrology (Carlton) 2015;20:352-9.

30. Jensen PT, Karnes J, Jones K, et al. Quantitative evaluation of a pediatric rheumatology transition program. Pediatr Rheumatol 2015:13:17.

31. Suris JC, Akre C. Key elements for, and indicators of, a successful transition: an international Delphi study. J Adolesc Health 2015;56:612-18.

32. Watson R, Parr JR, Joyce C, et al. Models of transitional care for young people with complex health needs: a scoping review. Child Care Health Dev 2011;37:780-91.

33. Nakhla M, Daneman D, Frank M, et al. Translating transition: a critical review of the diabetes literature. J Pediatr Endocrinol Metab 2008;21:507-16.

34. Prior $\mathrm{M}$, McManus $\mathrm{M}$, White $\mathrm{P}$, et al. Measuring the "triple aim" in transition care: a systematic review. Pediatrics 2014;134:e1648-1661.

35. Ovretveit J. Understanding the conditions for improvement: research to discover which context influences affect improvement success. BMJ Qual Saf 2011;20(Suppl 1):i18-23.

36. Barlow J, Wright C, Sheasby J, et al. Self-management approaches for people with chronic conditions: a review. Patient Educ Couns 2002;48:177-87.

37. Tariq S, Woodman J. Using mixed methods in health research JRSM Short Rep 2013:4:2042533313479197.

38. van Hooft S, van der Knaap L, Latour J, et al. Wat zijn de huidige werkwijzen rondom de ondersteuning van zelfmanagement in de zorg voor jongeren met een chronische aandoening binnen het Erasmus MC-Sophia? Rotterdam: Hogeschool Rotterdam, Kenniscentrum Zorginnovatie, 2012.

39. Wilkinson D, Robinson AJ. You're Welcome quality criteria: making health services young-people-friendly. Sex Transm Infect 2009;85:233-4.
40. Hargreaves DS. Revised You're Welcome criteria and future developments in adolescent healthcare. J Clin Res Pediatr Endocrinol 2011;3:43-50

41. Shaw K, Southwood T, McDonagh J. Development and preliminary validation of the "Mind the Gap" scale to assess satisfaction with transitional health care among adolescents with juvenile idiopathic arthritis. Child Care Health Dev 2007:33:380-8.

42. Sonneveld H, Strating M, Van Staa A, et al. Gaps in transitional care: what are the perceptions of adolescents, parents and providers? Child Care Health Dev 2013;39:69-80.

43. Nehring WM, Betz CL, Lobo ML. Uncharted territory: systematic review of providers' roles, understanding, and views pertaining to health care transition. J Pediatr Nurs 2015;30:732-47.

44. Colver AF, Merrick H, Deverill M, et al. Study protocol: longitudinal study of the transition of young people with complex health needs from child to adult health services. BMC Public Health 2013;13:675.

45. Sharma N, O'Hare K, Antonelli RC, et al. Transition care: future directions in education, health policy, and outcomes research. Acad Pediatr 2014;14:120-7.

46. Tsybina I, Kingsnorth S, Maxwell J, et al. Longitudinal Evaluation of Transition Services ("LETS Study"): protocol for outcome evaluation. BMC Pediatr 2012;12:51.

47. Fair C, Cuttance J, Sharma N, et al. International and interdisciplinary identification of health care transition outcomes. JAMA Paediatr 2015;30:1-7.

48. Woodward JF, Swigonski NL, Ciccarelli MR. Assessing the health, functional characteristics, and health needs of youth attending a noncategorical transition support program. $J$ Adolesc Health 2012:51:272-8.

49. Simmonds NJ, Macneill SJ, Cullinan P, et al. Cystic fibrosis and survival to 40 years: a case-control study. Eur Respir $J$ 2010;36:1277-83.

50. Tuchman L, Schwartz M. Health outcomes associated with transition from pediatric to adult cystic fibrosis care. Pediatrics 2013;132:847-53.

51. Goodhand JR, Kamperidis N, Sirwan B, et al. Factors associated with thiopurine non-adherence in patients with inflammatory bowel disease. Aliment Pharmacol Ther 2013;38:1097-108.

52. Leung Y, Heyman MB, Mahadevan U. Transitioning the adolescent inflammatory bowel disease patient: guidelines for the adult and pediatric gastroenterologist. Inflamm Bowel Dis 2011;17:2169-73.

53. Bryant RV, Trott MJ, Bennett A, et al. Transition of care in inflammatory bowel disease: mind the gap! Theory, practice and recommendations for an Australian context. Intern Med J 2013;43:1171-6.

54. Goodhand J, Hedin CR, Croft NM, et al. Adolescents with IBD: the importance of structured transition care. J Crohns Colitis 2011:5:509-19.

55. Godbout A, Tejedor I, Malivoir S, et al. Transition from pediatric to adult healthcare: assessment of specific needs of patients with chronic endocrine conditions. Horm Res Paediatr 2012;78:247-55.

56. Bollegala N, Brill H, Marshall JK. Resource utilization during pediatric to adult transfer of care in IBD. J Crohns Colitis 2013;7:e55-60.

57. Gleeson H, Davis J, Jones J, et al. The challenge of delivering endocrine care and successful transition to adult services in adolescents with congenital adrenal hyperplasia: experience in a single centre over 18 years. Clin Endocrinol (Oxf) 2013;78:23-8.

58. Quon BS, Aitken ML. Cystic fibrosis: what to expect now in the early adult years. Paediatr Respir Rev 2012;13:206-14.

59. Simmonds NJ, Cullinan P, Hodson ME. Growing old with cystic fibrosis - the characteristics of long-term survivors of cystic fibrosis. Respir Med 2009;103:629-35.

60. Okumura MJ, Ong T, Dawson D, et al. Improving transition from paediatric to adult cystic fibrosis care: programme implementation and evaluation. BMJ Qual Saf 2014;23(Suppl 1):i64-72.

61. Zhang LF, Ho JS, Kennedy SE. A systematic review of the psychometric properties of transition readiness assessment tools in adolescents with chronic disease. BMC Pediatr 2014;14:4.

62. Simmonds NJ. Ageing in cystic fibrosis and long-term survival. Paediatr Respir Rev 2013;14(Suppl 1):6-9.

63. Suri $R$, Metcalfe $C$, Wallis $C$, et al. Assessing the usefulness of outcomes measured in a cystic fibrosis treatment trial. Respir Med 2007;101:254-60.

64. Doull I. Cystic fibrosis papers of the year 2012. Paediatr Respir Rev 2013;14(Suppl 1):28-30.

65. Hodson ME, Simmonds NJ, Warwick WJ, et al. An international/ multicentre report on patients with cystic fibrosis (CF) over the age of 40 years. J Cyst Fibros 2008;7:537-42.

66. Dabadie A, Troadec F, Heresbach D, et al. Transition of patients with inflammatory bowel disease from pediatric to adult care. Gastroenterol Clin Biol 2008;32:451-9. 
67. Kirk J, Clayton P. Specialist services and transitional care in paediatric endocrinology in the UK and Ireland. Clin Endocrinol (Oxf) 2006;65:59-63.

68. Bloom SR, Kuhlthau K, Van Cleave J, et al. Health care transition for youth with special health care needs. J Adolesc Health 2012;51:213-19.

69. McLaughlin SE, Diener-West M, Indurkhya A, et al. Improving transition from pediatric to adult cystic fibrosis care: lessons from a national survey of current practices. Pediatrics 2008;121: e1160-1166.

70. Escher JC. Transition from pediatric to adult health care in inflammatory bowel disease. Dig Dis 2009;27:382-6.

71. Bishop J, Lemberg DA, Day A. Managing inflammatory bowel disease in adolescent patients. Adolesc Health Med Ther 2014;5:1-13.

72. Stanhope R. Transition from paediatric to adult endocrinology: hypopituitarism. Growth Horm IGF Res 2004;14(Suppl A):S85-88.

73. Wensing $M$, van Lieshout J, Jung HP, et al. The Patients Assessment Chronic IIIness Care (PACIC) questionnaire in The Netherlands: a validation study in rural general practice. BMC Health Serv Res 2008;8:182.

74. Glasgow RE, Wagner EH, Schaefer J, et al. Development and validation of the Patient Assessment of Chronic Illness Care (PACIC). Med Care 2005;43:436-44.

75. Battersby MW, Ask A, Reece MM, et al. The Partners in Health scale: The development and psychometric properties of a generic assessment scale for chronic condition self-management. Aust J Prim Health 2003;9:41-52.

76. Petkov J, Harvey $P$, Battersby M. The internal consistency and construct validity of the partners in health scale: validation of a patient rated chronic condition self-management measure. Qual Life Res 2010;19:1079-85.

77. Strating MMH, Cramm JM, Sonneveld HM, et al. Betere transitiezorg voor jongeren met chronische aandoeningen. Evaluatieonderzoek van het Actieprogramma Op Eigen Benen Vooruit! [Better transitional care for young people with chronic conditions. Evaluation of the Action 'Program On Your Own Feet!']. Rotterdam: Erasmus University Rotterdam, Institute of Health Policy \& Management, 2013.

78. El-Matary W. Transition of children with inflammatory bowel disease: big task, little evidence. World J Gastroenterol 2009;15:3744-7.

79. Fishman LN, Barendse RM, Hait E, et al. Self-management of older adolescents with inflammatory bowel disease: a pilot study of behavior and knowledge as prelude to transition. Clin Pediatr (Phila) 2010:49:1129-33.

80. Van Staa AL. On Your Own Feet: Adolescents with chronic conditions and their preferences and competencies for care (Doctoral dissertation). Rotterdam: Rotterdam University, 2012.

81. Morton AM. Symposium 6: Young people, artificial nutrition and transitional care. The nutritional challenges of the young adult with cystic fibrosis: transition. Proc Nutr Soc 2009;68:430-40.

82. Desir B, Seidman EG. Transitioning the paediatric IBD patient to adult care. Best Pract Res Clin Gastroenterol 2003;17:197-212.

83. Mahler C, Hermann K, Horne R, et al. Assessing reported adherence to pharmacological treatment recommendations. Translation and evaluation of the Medication Adherence Report Scale (MARS) in Germany. J Eval Clin Pract 2010;16:574-9.

84. Bent N, Tennant A, Swift T, et al. Team approach versus ad hoc health services for young people with physical disabilities: a retrospective cohort study. Lancet 2002;360:1280-6.

85. Limperg PF, Haverman L, van Oers HA, et al. Health related quality of life in Dutch young adults: psychometric properties of the PedsQL generic core scales young adult version. Health Qual Life Outcomes 2014:12:9.

86. Thomas DR. A general inductive approach for analyzing qualitative evaluation data. Am J Eval 2006;27:237-46.

87. Levy-Shraga Y, Elisha N, Ben-Ami M, et al. Glycemic control and clinic attendance of emerging adults with type 1 diabetes at a transition care clinic. Acta Diabetol 2016;53:27-33.

88. Jurasek L, Ray L, Quigley D. Development and implementation of an adolescent epilepsy transition clinic. J Neurosci Nurs 2010;42:181-9.

89. Geerlings RP, Aldenkamp AP, de With PH, et al. Transition to adult medical care for adolescents with epilepsy. Epilepsy Behav 2015;44:127-35.

90. Blum RW, Garell D, Hodgman $\mathrm{CH}$, et al. Transition from child-centered to adult health-care systems for adolescents with chronic conditions: a position paper of the Society for Adolescent Medicine. J Adolesc Health 1993;14:570-6.

91. Steinbeck KS, Shrewsbury VA, Harvey V, et al. A pilot randomized controlled trial of a post-discharge program to support emerging adults with type 1 diabetes mellitus transition from pediatric to adult care. Pediatr Diabet 2015;16:634-9.

92. Nieboer AP, Cramm JM, Sonneveld HM, et al. Reducing bottlenecks: professionals' and adolescents' experiences with transitional care delivery. BMC Health Serv Res 2014;14:47. 